\title{
3.
}

\section{Quantification of glucocorticoid receptors cDNA through real time PCR in Dicentrarchus labrax following a stress simulating experiment}

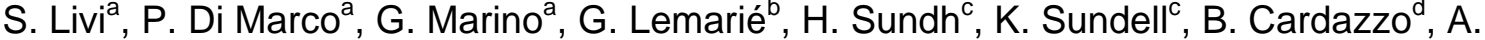 \\ Mininnid and T. Patarnello ${ }^{d}$
}

\author{
a ICRAM Central Institute for Marine Research, Rome, Italy \\ ${ }^{b}$ IFREMER, Palavàs-les-Flots, France \\ ${ }^{c}$ Department of Zoology, University of Göteborg, Sweden \\ d Department of Public Health, Comparative Pathology and Veterinary Hygiene, University of Padua, Italy
}

\begin{abstract}
Glucocorticoid receptors (GRs) are transcriptional factors activated by cortisol that regulate several physiological functions in vertebrates (metabolism, immune response, growth) and play a major role in the stress response process. In this study stress conditions have been simulated through a exogenous cortisol challenge in Dicentrarchus labrax and gene expression of both glucocorticoid receptors assessed after 7 and 21 days. Main objectives of this study are the investigation of candidate stress indicator genes for aquaculture and knowledge gain of the GR gene expression regulation in response to plasma cortisol level. Complete cDNA sequences of sea bass GRs and the housekeeping gene $\beta$ actin were retrieved from GenBank (AY549305, AY619996, AY148350) to develop primers and probes for real time PCR. GRs mRNA levels between control and treated cortisol groups within each sampling time were not statistically different (pN0.05), whereas higher concentrations of cortisol and glucose were observed after 7 days. After 21 days the GR61 mRNA average level was lower in cortisol-treated samples with $p=0.06$. The result can be compared with the one previously obtained in the pilot experiment in which an exogenous cortisol dose of $50 \mathrm{mg} / \mathrm{g}$ elicited a significant decrease $(p=0.01)$ of GR61 mRNA after 21 days from implantation. In this study the response of both GR genes to a stress simulating factor was investigated for the first time in D. labrax thus adding further information to the still unclear molecular pattern of GRs response to cortisol in fish species.
\end{abstract}

